\title{
On The Anti-Synchronization Of Fractional-Order Chaotic And Hyperchaotic Systems Via Modified Adaptive Sliding-Mode Control
}

\author{
A .Othman Almatroud ${ }^{1}$, Y. Jawarneh ${ }^{1}$, Israr Ahmad ${ }^{2}$, M. Mossa Al-sawalha ${ }^{1}$, M. S. M. Noorani ${ }^{3}$ \\ ${ }^{1}$ Department of Mathematics, Faculty of Science, University of Ha'il, Kingdom of Saudi Arabia. \\ 2 Department of General Requirements, College of Applied Sciences Nizwa, Oman. \\ ${ }^{3}$ School of Mathematical Sciences, Universiti Kebangsaan Malaysia, 43600 Bangi, Selangor, Malaysia.
}

Article History: Received: 11 January 2021; Accepted: 27 February 2021; Published online: 5 April 2021

\begin{abstract}
This paper investigates the anti-synchronization problem between two different fractional-order chaotic and hyperchaotic systems using the modified adaptive sliding mode control technique in the presence of uncertain system parameters. To construct the proposed scheme, a simple sliding surface is first designed. Then, the modified adaptive sliding-mode controller is derived to guarantee the occurrence of sliding motion. Based on the Lyapunov stability theory, the adaptive controllers with corresponding parameter update laws are designed such that the different chaotic and hyperchaotic systems can be anti-synchronized asymptotically. Finally, numerical simulations are presented to demonstrate the efficiency of the proposed anti-synchronization scheme.
\end{abstract}

Key words: Sliding-mode controller; Anti-synchronization; Fractional-order chaotic systems.

\section{Introduction}

Fractional-order chaotic systems have recently received considerable attention for their interdisciplinary nature, being manifest in diverse areas of research including dielectric polarization, electrode-electrolyte polarization, electromagnetic waves, viscoelastic systems, quantitative finance, bioengineering, diffusion waves, and nuclear magnetic resonance. Major recent topics of interest for nonlinear-science applications include the synchronization and anti-synchronization of fractionalorder chaotic systems in a broad variety of situations and the use of such systems for various purposes. These topics require a knowledge of basic mathematical properties of fractional-order chaotic systems combined with specific practical considerations of various applications (Yang, 2012, Al-sawalha, 2016, Hajipour and Aminabadi, 2016, Al-sawalha, 2017).

Several important and fundamental results have been reported with regard to synchronization and anti-synchronization. Various powerful methods of chaos synchronization and anti-synchronization for fractional-order dynamical systems have also been proposed. These include, e.g., feedback (Deepika, Sandeep \& Shiv,2018), active control(Tsung, Tun \& Valentina 2011), Q-S synchronization (Ardashir, Sehraneh, Okyay and Sohrab 2019), adaptive synchronization (Ardashir \& Sehraneh, 2017, Ardashir \& Sehraneh, 2018), and projective synchronization (Sakthivel, Sakthivel, Nithya, Selvaraj and Kwon, 2018). In addition to chaos synchronization, the antisynchronization of fractional-order chaotic systems is a fascinating concept that has recently attracted considerable interest among nonlinear scientists. Chaos anti-synchronization involves two fractional-order chaotic systems, namely the master and slave systems. Anti-synchronization controllers are designed to give the state vectors of synchronized systems the same amplitude but opposite signs to those of the driving system. Therefore, the sum of two signals is expected to converge to zero when anti-synchronization appears in either the synchronized or driving system .

For fractional-order chaos anti-synchronization, unknown model uncertainties have an adverse effect on anti-synchronization behavior, leading to a decrease in the performance of real systems. Scientific investigations into anti-synchronization in fractional-order chaotic systems with different kinds of uncertainties have addressed this challenge by several approaches to system control (Selvaraj, Kwon \& Sakthivel, 2019, Agrawal, \& Das 2013, Pourmahmood, Khanmohammadi \& Alizadeh 2011).

One effective method for dealing with uncertainties is sliding-mode control, which has the advantages of a fast dynamic response and a low sensitivity to external disturbances and model uncertainties. Many important results have been reported in the literature (Yahyazadeh, Noei \& Ghaderi, 2011, Chen, Park, Cao \& Qiu,2017, Li, 2012, Pourmahmood \& Heydari, 2012). The design for adaptive sliding-mode controller combines an adaptive controller and a sliding-mode controller. During the design process, the determination of some of the controller parameters is somewhat arduous. The main purpose of the present work is to introduce a new modification of the adaptive 
sliding mode for the purpose of achieving anti-synchronization in different fractional- and integerorder chaotic systems in the presence of fully unknown parameters, in both the master and slave chaotic systems. A simple sliding surface, suitable for this purpose, which includes antisynchronization errors, is constructed. Appropriate update laws are derived to determine the unknown parameters. The stability and robustness of the proposed modified adaptive sliding mode is proved using Lyapunov stability theory. Finally, two simulation examples are provided to demonstrate the effectiveness of the proposed anti-synchronization scheme.

\section{Properties of fractional derivative}

Fractional calculus is a generalization of integration and differentiation to a non-integer-order integrodifferential operator ${ }_{a} D_{t}^{\alpha}$ defined by

$$
{ }_{a} D_{t}^{\alpha}=\left\{\begin{array}{lc}
\frac{\mathrm{d}^{\alpha}}{\mathrm{dt} \mathrm{t}^{\alpha}} & \alpha>0, \\
1 & \alpha=0, \\
\int_{\mathrm{a}}^{\mathrm{t}}(\mathrm{d} \tau)^{-\alpha} & \alpha<0,
\end{array}\right.
$$

In this work, we adopts the Riemann-Liouvile definition ( Podlubny, 1999, Agrawal, \& Das 2013), which is defined by

$$
{ }_{a} D_{t}^{\alpha} x(t)=\frac{d^{n}}{d^{n}} n_{t}^{n-\alpha} x(t), \quad \alpha>0,
$$

where $\mathrm{n}=\lceil\alpha\rceil$, i.e., $\mathrm{n}$ is the first integer which is not less than $\alpha . \mathrm{J}^{\varphi}$ is the fractional Riemann-Liouville integral integral operator which is described as follows:

$$
J_{\mathrm{t}}^{9} \varphi(\mathrm{t})=\frac{1}{\Gamma(\vartheta)} \int_{0}^{\mathrm{t}} \frac{\varphi(v)}{(\mathrm{t}-\mathrm{v})^{1-9}} d v,
$$

with $0<\vartheta \leq 1, \Gamma($.$) is the gamma function. For \mathrm{s}, \mathrm{n} \geq 0$, there exist integers $\alpha$ and $\beta$ such that $0 \leq \alpha-1 \leq$ $\mathrm{s}<\alpha$, and $0 \leq \beta-1 \leq \mathrm{n}<\beta$. Then,

$$
{ }_{a} D_{t}^{s}\left({ }_{a} D_{t}^{n} x(t)\right)={ }_{a} D_{t}^{s+n} x(t)-\sum_{j=1}^{n}\left[{ }_{a} D_{t}^{n-j} x(t)\right]_{t=a} \frac{(t-a)^{-s-j}}{\Gamma(1-s-j)} .
$$

For $\mathrm{s}>n \geq 0, \alpha$ and $\beta$ are integers such that $0 \leq \alpha-1 \leq \mathrm{s}<\alpha$, and $0 \leq \beta-1 \leq \mathrm{n}<\beta$. Then,

$$
{ }_{A} D_{t}^{s}\left({ }_{a} D_{t}^{-n} x(t)\right)={ }_{a} D_{t}^{s-n} x(t) .
$$

\section{Anti-synchronization of fractional order chaos using the modified adaptive sliding-mode control method}

Given the fractional order drive system of the form

$$
D_{t}^{p} x_{d}=f\left(x_{d}\right)+F\left(x_{d}\right) \varphi,
$$

where $x_{d}=\left(x_{d 1}, x_{d 2}, \ldots, x_{d n}\right) \in R^{n}, f: R^{n} \rightarrow R^{n}$ is a continuous vector function, $F: R^{n} \rightarrow R^{n \times d}$ is a matrix function, and $\varphi \in \mathrm{R}^{\mathrm{d}}$ is the unknown parameter vectors. Let the corresponding response system be

$$
D_{\mathrm{t}}^{\mathrm{p}} \mathrm{y}_{\mathrm{r}}=\mathrm{g}\left(\mathrm{y}_{\mathrm{r}}\right)+\mathrm{G}\left(\mathrm{y}_{\mathrm{r}}\right) \psi+\mathrm{u},
$$

where $y_{r}=\left(y_{r 1}, y_{r 2}, \ldots, y_{r n}\right) \in R^{n}, g: R^{n} \rightarrow R^{n}$ is a continuous function, $G: R^{n} \rightarrow R^{n \times k}$ is a matrix function, $\psi \in \mathrm{R}^{\mathrm{k}}$ is the unknown parameter vectors, and $\mathrm{u} \in \mathrm{R}^{\mathrm{n}}$ is the control input. The controlled resulting antisynchronisation error system can be expressed by the following dynamical system

$$
D_{t}^{p} \mathrm{e}(\mathrm{t})=\mathrm{g}\left(\mathrm{y}_{\mathrm{r}}\right)+\mathrm{G}\left(\mathrm{y}_{\mathrm{r}}\right) \psi+\mathrm{f}\left(\mathrm{x}_{\mathrm{d}}\right)+\mathrm{F}\left(\mathrm{x}_{\mathrm{d}}\right) \varphi+\mathrm{u},
$$

Our goal is to introduce an modified adaptive sliding-mode procedure to design the controller $u$ to make the controlled uncertain response system anti-synchronous with master system asymptotically, such that

$$
\lim _{t \rightarrow \infty}\|e\|=\lim _{t \rightarrow \infty}\left\|y_{r}\left(t, y_{0}\right)+x_{d}\left(t, x_{0}\right)\right\|=0,
$$


In accordance with the design procedure used for an modified adaptive sliding-mode control, if the nonlinear control function $\mathrm{u}$ is selected in (7) as follows:

$$
\begin{aligned}
\mathrm{u}= & -\mathrm{f}\left(\mathrm{x}_{\mathrm{d}}\right)+\mathrm{F}\left(\mathrm{x}_{\mathrm{d}}\right) \varphi-\mathrm{g}\left(\mathrm{y}_{\mathrm{r}}\right)+\mathrm{G}\left(\mathrm{y}_{\mathrm{r}}\right) \psi+\mathrm{D}_{\mathrm{t}}^{\mathrm{p}-1}\left[-\mathrm{F}\left(\mathrm{x}_{\mathrm{d}}\right)(\widehat{\varphi}-\varphi)-\mathrm{G}\left(\mathrm{y}_{\mathrm{r}}\right)(\widehat{\psi}-\psi)\right. \\
& \left.-\left(\mathrm{D}_{\mathrm{t}}^{\mathrm{p}-1} \mathrm{e}(\mathrm{t})\right) \frac{(\mathrm{t})^{-(\mathrm{p}-1)-1}}{\Gamma(-(\mathrm{p}-1))}-\mathrm{w}(\mathrm{t}) \mathrm{K}\right],
\end{aligned}
$$

where $\widehat{\varphi}, \widehat{\psi}$ are estimate values of the unknown parameters and $\mathrm{k}=\left[\mathrm{k}_{1}, \mathrm{k}_{2}, \ldots, \mathrm{k}_{\mathrm{n}}\right]^{\mathrm{T}}$ is a constant gain vector.

Now, substituting u into the anti-synchronization error system (8) yields a form that is comfortable for the oncoming stability analysis:

$$
D_{t}^{p} e(t)=D_{t}^{p-1}\left[-F\left(x_{d}\right)(\widehat{\varphi}-\varphi)-G\left(y_{r}\right)(\widehat{\psi}-\psi)-\left(D_{t}^{p-1} e(t)\right) \frac{(t)^{-(p-1)-1}}{\Gamma(-(p-1))}-w(t) K\right] .
$$

Here $\mathrm{w}(\mathrm{t}) \in \mathrm{R}$ is a control input and can be determined as

$$
w(t) \begin{cases}w^{+}(t) & s(e) \geq 0 \\ w^{-}(t) & s(e)<0\end{cases}
$$

where $s=s(e)$ is a switching surface which introduces the desired sliding dynamics. The sliding surface function is designed as

$$
\mathrm{s}(\mathrm{e})=\mathrm{ce},
$$

where $\mathrm{c}=\left[\mathrm{c}_{1}, \mathrm{c}_{2}, \ldots, \mathrm{c}_{\mathrm{n}}\right]$ is a constant vector. A necessary two conditions for the state trajectory to fulfilled on the sliding surface:

$$
\mathrm{s}(\mathrm{e})=0 \quad \text { together with } \dot{\mathrm{s}}(\mathrm{e})=0 .
$$

The second condition is a necessary condition to constrain the state trajectory to stay on the switching surface $\mathrm{s}(\mathrm{e})=0$. In accordance to the to sliding-mode design strategy, we design the the sliding mode as follows

$$
\mathrm{w}(\mathrm{t})=\left[\frac{\mathrm{s}}{|s|+\gamma}\right],
$$

where $\gamma>0$. The update laws parameters are defined as

$$
\begin{aligned}
& \dot{\hat{\alpha}}=\left[\mathrm{F}\left(\mathrm{x}_{\mathrm{d}}\right)\right]^{\mathrm{T}} \lambda, \\
& \dot{\hat{\beta}}=\left[\mathrm{G}\left(\mathrm{y}_{\mathrm{r}}\right)\right]^{\mathrm{T}} \lambda,
\end{aligned}
$$

where $\lambda=\mathrm{sc}^{\mathrm{T}}$.

Theorem. 1. Considering the error dynamic systems (11) with control laws (10) that obeys update laws parameters in (16). Then the error dynamic systems trajectories will converge to the sliding surface $s(t)=0$.

Proof. Consider the following Lyapunov candidate function:

$$
\mathrm{V}=\frac{1}{2}\left[\mathrm{~s}^{2}+\widetilde{\varphi}^{\mathrm{T}} \widetilde{\varphi}+\widetilde{\psi}^{\mathrm{T}} \widetilde{\psi}\right]
$$

where $\widetilde{\varphi}=\widehat{\varphi}-\varphi$ and $\widetilde{\psi}=\widehat{\psi}-\psi$. The time derivative of (17) is

$$
\dot{V}=\left[s \dot{e}^{\mathrm{T}} \mathrm{c}^{\mathrm{T}}+\widetilde{\varphi}^{\mathrm{T}} \dot{\widetilde{\varphi}}+\widetilde{\psi}^{\mathrm{T}} \dot{\widetilde{\psi}}\right] .
$$

Using (4) in (18), yields $\dot{V}$ as:

$$
\dot{\mathrm{V}}=\mathrm{s}\left[D_{\mathrm{t}}^{\mathrm{q}-1}\left(D_{\mathrm{t}}^{\mathrm{q}} \mathrm{e}\right)+\left(D_{\mathrm{t}}^{\mathrm{q}-1} \mathrm{e}(\mathrm{t})\right) \frac{(\mathrm{t})^{-(\mathrm{q}-1)-1}}{\Gamma(-(\mathrm{q}-1))}\right]^{\mathrm{T}} \mathrm{c}^{\mathrm{T}}+\widetilde{\varphi}^{\mathrm{T}} \dot{\tilde{\varphi}}+\widetilde{\psi}^{\mathrm{T}} \dot{\widetilde{\psi}} .
$$

From (11) and (18), we obtain 


$$
\begin{aligned}
\dot{\mathrm{V}}= & \mathrm{s}\left[\mathrm { D } _ { \mathrm { t } } ^ { \mathrm { q } - 1 } \left(\mathrm { D } _ { \mathrm { t } } ^ { \mathrm { q } - 1 } \left[-\mathrm{F}\left(\mathrm{x}_{\mathrm{d}}\right) \widetilde{\varphi}-\mathrm{G}\left(\mathrm{y}_{\mathrm{r}}\right) \widetilde{\psi}-\left(\mathrm{D}_{\mathrm{t}}^{\mathrm{q}-1} \mathrm{e}(\mathrm{t})\right) \frac{(\mathrm{t})^{-(\mathrm{q}-1)-1}}{\Gamma(-(\mathrm{q}-1))}\right.\right.\right. \\
& \left.\left.\left.-\frac{\mathrm{s}}{|\mathrm{s}|+\gamma} \mathrm{k}\right]\right)+\left(\mathrm{D}_{\mathrm{t}}^{\mathrm{q}-1} \mathrm{e}(\mathrm{t})\right) \frac{(\mathrm{t})^{-(\mathrm{q}-1)-1}}{\Gamma(-(\mathrm{q}-1))}\right]^{\mathrm{T}} \mathrm{c}^{\mathrm{T}}+\widetilde{\varphi}^{\mathrm{T}} \dot{\widetilde{\varphi}}+\widetilde{\Psi}^{\mathrm{T}} \dot{\widetilde{\psi}}
\end{aligned}
$$

since $\forall \mathrm{q} \in[0,1],(1-\mathrm{q})>0$ and $(\mathrm{q}-1)<0$. Now, using $(5)$ and $(16),(20)$ reduces to

$$
\begin{aligned}
\dot{\mathrm{V}}= & \mathrm{s}\left[\left(-\mathrm{F}\left(\mathrm{x}_{\mathrm{d}}\right) \widetilde{\varphi}-\mathrm{G}\left(\mathrm{y}_{\mathrm{r}}\right) \widetilde{\Psi}-\left(\mathrm{D}_{\mathrm{t}}^{\mathrm{q}-1} \mathrm{e}(\mathrm{t})\right) \frac{(\mathrm{t})^{-(\mathrm{q}-1)-1}}{\Gamma(-(\mathrm{q}-1))}-\frac{\mathrm{s}}{|\mathrm{s}|+\gamma} \mathrm{k}\right)\right. \\
& \left.+\left(\mathrm{D}_{\mathrm{t}}^{\mathrm{q}-1} \mathrm{e}(\mathrm{t})\right) \frac{(\mathrm{t})^{-(\mathrm{q}-1)-1}}{\Gamma(-(\mathrm{q}-1))}\right]^{\mathrm{T}} \mathrm{c}^{\mathrm{T}}+\widetilde{\varphi}^{\mathrm{T}} \dot{\widetilde{\varphi}}+\widetilde{\Psi}^{\mathrm{T}} \dot{\widetilde{\Psi}} \\
\dot{\mathrm{V}}= & \mathrm{s}\left[-\mathrm{F}\left(\mathrm{x}_{\mathrm{d}}\right) \widetilde{\varphi}-\mathrm{G}\left(\mathrm{y}_{\mathrm{r}}\right) \widetilde{\Psi}-\frac{\mathrm{s}}{|\mathrm{s}|+\gamma} \mathrm{k}\right]^{\mathrm{T}} \mathrm{c}^{\mathrm{T}}+\widetilde{\varphi}^{\mathrm{T}} \mathrm{F}\left(\mathrm{x}_{\mathrm{d}}\right)^{\mathrm{T}} \lambda+\widetilde{\Psi}^{\mathrm{T}} \mathrm{G}\left(\mathrm{y}_{\mathrm{r}}\right)^{\mathrm{T}} \lambda .
\end{aligned}
$$

Then, (22) yields

$$
\dot{\mathrm{V}}=-\operatorname{ck}\left[\frac{\mathrm{s}^{2}}{|\mathrm{~s}|+\gamma}\right]<0 .
$$

Since $s^{2}>0$ and $|s|>0$ both hold true, then, when $\mathrm{e} \neq 0$ and ck $>0$, the inequality $\dot{V}<0$ holds. According to the Lyapunov stability theory (Liapunov, 1966) $V$ is positive-definite, and $\dot{V}$ is negative-definite. Thus, the trajectories of the fractional error dynamical system (8) asymptotically converge to $s(t)=0$. Therefore, the state variables of the of the drive system (6) and the states variables of the response (7) system can be antisynchronized asymptotically and globally with the control law (10) and the adaptive parameter update laws (16). Here, the proof is completed.

\section{Modified adaptive sliding mode anti-synchronization of two fractional order chaotic systems}

To observe anti-synchronization behavior between two different fractional order chaotic systems by adaptive sliding-mode control, the drive system is assumed to be a fractional-order Lorenz system (Zhou, \& Zhu,2011), and a fractional-order Chen system (Lu \& Chen, 2006), is considered as the response system. The drive system is described as

$$
\begin{aligned}
& \mathrm{D}_{\mathrm{t}}^{\mathrm{q}_{1} \mathrm{x}_{1}}=\mathrm{a}_{1}\left(\mathrm{y}_{1}-\mathrm{x}_{1}\right), \\
& \mathrm{D}_{\mathrm{t}}^{\mathrm{q}_{2}} \mathrm{y}_{1}=\mathrm{b}_{1} \mathrm{x}_{1}-\mathrm{x}_{1} \mathrm{z}_{1}-\mathrm{y}_{1}, \\
& \mathrm{D}_{\mathrm{t}}^{\mathrm{q}_{3}} \mathrm{z}_{1}=\mathrm{x}_{1} \mathrm{y}_{1}-\mathrm{c}_{1} \mathrm{z}_{1},
\end{aligned}
$$

and the response system as

$$
\begin{aligned}
& D_{t}^{q_{1}} x_{2}=a_{2}\left(y_{2}-x_{2}\right)+u_{1}, \\
& D_{t}^{q_{2}} y_{2}=\left(b_{2}-a_{2}\right) x_{2}-x_{2} z_{2}+b_{2} y_{2}+u_{2}, \\
& D_{t}^{q_{3}} z_{2}=x_{2} y_{2}-c_{2} z_{2}+u_{3},
\end{aligned}
$$

where the variables $\left(\mathrm{u}_{1}, \mathrm{u}_{2}, \mathrm{u}_{3}\right)^{\mathrm{T}}$ are controllers to be designed. Let $\mathrm{e}_{1}=\mathrm{x}_{2}+\mathrm{x}_{1}, \mathrm{e}_{2}=\mathrm{y}_{2}+\mathrm{y}_{1}, \mathrm{e}_{3}=\mathrm{z}_{2}+\mathrm{z}_{1}$. Then, we get the following error dynamic system between the drive (24) and response (24) systems

$$
\begin{aligned}
& D_{t}^{q_{1}} e_{1}(t)=a_{2}\left(y_{2}-x_{2}\right)+a_{1}\left(y_{1}-x_{1}\right)+u_{1}, \\
& D_{t}^{q_{2}} e_{2}(t)=\left(b_{2}-a_{2}\right) x_{2}-x_{2} z_{2}+b_{2} y_{2}+b_{1} x_{1}-x_{1} z_{1}-y_{1}+u_{2} \\
& D_{t}^{q_{3}} e_{3}(t)=x_{2} y_{2}-c_{2} z_{2}+x_{1} y_{1}-c_{1} z_{1}+u_{3} .
\end{aligned}
$$

The goal of the modified adaptive sliding-mode control is to find an effective controller function $\left(\mathrm{u}_{1}, \mathrm{u}_{2}, \mathrm{u}_{3}\right)^{\mathrm{T}}$ capable anti-synchronizing the states of the response and drive systems with a parameter estimation update law. An appropriate sliding surface can be chosen as

$$
\begin{aligned}
& s(e)=e_{1}+e_{2}-e_{3}, \\
& w(t)=\frac{s}{|s|+0.01},
\end{aligned}
$$


It is assumed that the constant vectors are $\mathrm{c}=(1,1,-1), \mathrm{k}=(5,10,0)^{\mathrm{T}}$ and $\gamma=0.01$. The adaptive slidingmode controller of the error dynamic system (26) can be calculated as follows

$$
\begin{aligned}
\mathrm{u}_{1}= & -\mathrm{a}_{2}\left(\mathrm{y}_{2}-\mathrm{x}_{2}\right)-\mathrm{a}_{1}\left(\mathrm{y}_{1}-\mathrm{x}_{1}\right)+D_{\mathrm{t}}^{\mathrm{q}_{1}-1}\left[-\hat{\mathrm{a}}_{2}\left(\mathrm{y}_{2}-\mathrm{x}_{2}\right)-\hat{\mathrm{a}}_{1}\left(\mathrm{y}_{1}-\mathrm{x}_{1}\right)-\left(\mathrm{D}_{\mathrm{t}}^{\mathrm{q}_{1}-1} \mathrm{e}_{1}(\mathrm{t})\right)\right. \\
& \left.\frac{(\mathrm{t})^{-\left(\mathrm{q}_{1}-1\right)-1}}{\Gamma\left(-\left(\mathrm{q}_{1}-1\right)\right)}-\frac{5 \mathrm{~s}}{|\mathrm{~s}|+0.01}\right], \\
\mathrm{u}_{2}= & -\left(\mathrm{b}_{2}-\mathrm{a}_{2}\right) \mathrm{x}_{2}+\mathrm{x}_{2} \mathrm{z}_{2}-\mathrm{b}_{2} \mathrm{y}_{2}-\mathrm{b}_{1} \mathrm{x}_{1}+\mathrm{x}_{1} \mathrm{z}_{1}+\mathrm{y}_{1}+D_{\mathrm{t}}^{\mathrm{q}_{2}-1}\left[-\left(\hat{\mathrm{b}}_{2}-\hat{\mathrm{a}}_{2}\right) \mathrm{x}_{2}-\hat{\mathrm{b}}_{2} \mathrm{y}_{2}\right. \\
& \left.-\hat{\mathrm{b}}_{1} \mathrm{x}_{1}-\left(\mathrm{D}_{\mathrm{t}}^{\mathrm{q}_{2}-1} \mathrm{e}_{2}(\mathrm{t})\right) \frac{(\mathrm{t})^{-\left(\mathrm{q}_{2}-1\right)-1}}{\Gamma\left(-\left(\mathrm{q}_{2}-1\right)\right)}-\frac{10 \mathrm{~s}}{|\mathrm{~s}|+0.01}\right], \\
\mathrm{u}_{3}= & -\mathrm{x}_{2} \mathrm{y}_{2}+\mathrm{c}_{2} \mathrm{z}_{2}-\mathrm{x}_{1} \mathrm{y}_{1}+\mathrm{c}_{1} \mathrm{z}_{1}+\mathrm{D}_{\mathrm{t}}^{\mathrm{q}_{3}-1}\left[\hat{\mathrm{c}}_{2} \mathrm{z}_{2}+\hat{\mathrm{c}}_{1} \mathrm{z}_{1}-\left(\mathrm{D}_{\mathrm{t}}^{\mathrm{q}_{3}-1} \mathrm{e}_{3}(\mathrm{t})\right) \frac{(\mathrm{t})^{-\left(\mathrm{q}_{3}-1\right)-1}}{\Gamma\left(-\left(\mathrm{q}_{3}-1\right)\right)}\right] .
\end{aligned}
$$

The adaptive laws for estimating the parameters $\hat{\mathrm{a}}_{1}, \hat{\mathrm{b}}_{1}, \hat{\mathrm{c}}_{1}, \hat{\mathrm{a}}_{2}, \hat{\mathrm{b}}_{2}$, and $\widehat{c}_{2}$ are chosen as follows:

$$
\begin{aligned}
& \dot{\tilde{\mathrm{a}}}_{1}=\mathrm{s}\left(\mathrm{y}_{1}-\mathrm{x}_{1}\right) \\
& \dot{\tilde{\mathrm{b}}}_{1}=\mathrm{sx}_{1} \\
& \dot{\tilde{\mathrm{c}}}_{1}=\mathrm{sz}_{1} \\
& \dot{\tilde{\mathrm{a}}}_{2}=\mathrm{s}\left(\mathrm{y}_{2}-\mathrm{x}_{2}\right)-\mathrm{s} \mathrm{x_{2 }} \\
& \dot{\tilde{\mathrm{b}}}_{2}=\mathrm{s}\left(\mathrm{x}_{2}+\mathrm{y}_{2}\right) \\
& \dot{\tilde{\mathrm{c}}}_{2}=\mathrm{sz}_{2}
\end{aligned}
$$

Theorem 2. The state variables of the of the drive system (24) and the states variables of the response (25) system can be anti-synchronized asymptotically and globally for all initial conditions using the control law (28) and the adaptive parameter update laws (29).

Proof. Substituting (28) into (26), this yields

$$
\begin{aligned}
& D_{t}^{q_{1}} e_{1}(t)=D_{t}^{q_{1}-1}\left[-\tilde{a}_{2}\left(y_{2}-x_{2}\right)-\tilde{a}_{1}\left(y_{1}-x_{1}\right)-\left(D_{t}^{q_{1}-1} e_{1}(t)\right) \frac{(t)^{-\left(q_{1}-1\right)-1}}{\Gamma\left(-\left(\mathrm{q}_{1}-1\right)\right)}-\frac{5 s}{|s|+0.01}\right], \\
& D_{t}^{q_{2}} e_{2}(t)=D_{t}^{q_{2}-1}\left[-\left(\tilde{b}_{2}-\tilde{a}_{2}\right) x_{2}-\tilde{b}_{2} y_{2}-\tilde{b}_{1} x_{1}-\left(D_{t}^{q_{2}-1} e_{2}(t)\right) \frac{(t)^{-\left(q_{2}-1\right)-1}}{\Gamma\left(-\left(\mathrm{q}_{2}-1\right)\right)}-\frac{10 s}{|s|+0.01}\right], \\
& D_{t}^{q_{3}} e_{3}(t)=D_{t}^{q_{3}-1}\left[\tilde{c}_{2} z_{2}+\tilde{c}_{1} z_{1}-\left(D_{t}^{q_{3}-1} e_{3}(t)\right) \frac{(t)^{-\left(q_{3}-1\right)-1}}{\Gamma\left(-\left(\mathrm{q}_{3}-1\right)\right)}\right],
\end{aligned}
$$

where $\tilde{a}_{1}=\hat{a}_{1}-a_{1}, \tilde{b}_{1}=\hat{b}_{1}-b_{1}, \tilde{c}_{1}=\hat{c}_{1}-c_{1}, \tilde{a}_{2}=\hat{a}_{2}-a_{2}, \tilde{b}_{2}=\hat{b}_{2}-b_{2}$, and $\tilde{c}_{2}=\hat{c}_{2}-c_{2}$. Selecting a Lyapunov function candidate in the form of

$$
\mathrm{V}=\frac{1}{2}\left(\mathrm{~s}^{2}+\tilde{\mathrm{a}}_{1}^{2}+\tilde{\mathrm{b}}_{1}^{2}+\tilde{\mathrm{c}}_{1}^{2}+\tilde{\mathrm{a}}_{2}^{2}+\tilde{\mathrm{b}}_{2}^{2}+\tilde{\mathrm{c}}_{2}^{2}\right) \text {. }
$$

Taking the derivative of (31) with respect to time using (4), one has

$$
\begin{aligned}
& \dot{\mathrm{V}}=\left(\mathrm{s} \dot{\mathrm{s}}+\tilde{\mathrm{a}}_{1} \dot{\tilde{\mathrm{a}}}_{1}+\tilde{\mathrm{b}}_{1} \dot{\tilde{\mathrm{b}}}_{1}+\tilde{\mathrm{c}}_{1} \dot{\tilde{\mathrm{c}}}_{1}+\tilde{\mathrm{a}}_{2} \dot{\tilde{\mathrm{a}}}_{2}+\tilde{\mathrm{b}}_{2} \dot{\tilde{\mathrm{b}}}_{2}+\tilde{\mathrm{c}}_{2} \dot{\tilde{\mathrm{c}}}_{2}\right) \\
& =s\left[D_{t}^{1-q_{1}}\left(D_{t}^{q_{1}} e_{1}(t)\right)+\left(D_{t}^{q_{1}-1} e_{1}(t)\right) \frac{(t)^{-\left(q_{1}-1\right)-1}}{\Gamma\left(-\left(q_{1}-1\right)\right)}\right]+s\left[D_{t}^{1-q_{2}}\left(D_{t}^{q_{2}} e_{2}(t)\right)+\left(D_{t}^{q_{2}-1} e_{2}(t)\right)\right. \\
& \left.\frac{(\mathrm{t})^{-\left(\mathrm{q}_{2}-1\right)-1}}{\Gamma\left(-\left(\mathrm{q}_{2}-1\right)\right)}\right]-\mathrm{s}\left[\mathrm{D}_{\mathrm{t}}^{1-\mathrm{q}_{3}}\left(\mathrm{D}_{\mathrm{t}}^{\mathrm{q}_{3}} \mathrm{e}_{3}(\mathrm{t})\right)+\left(\mathrm{D}_{\mathrm{t}}^{\mathrm{q}_{3}-1} \mathrm{e}_{3}(\mathrm{t})\right) \frac{(\mathrm{t})^{-\left(\mathrm{q}_{3}-1\right)-1}}{\Gamma\left(-\left(\mathrm{q}_{3}-1\right)\right)}\right]+\tilde{\mathrm{a}}_{1} \dot{\tilde{\mathrm{a}}}_{1}+\tilde{\mathrm{b}}_{1} \dot{\tilde{\mathrm{b}}}_{1}+\tilde{\mathrm{c}}_{1} \dot{\tilde{\mathrm{c}}}_{1} \\
& +\tilde{\mathrm{a}}_{2} \dot{\tilde{\mathrm{a}}}_{2}+\tilde{\mathrm{b}}_{2} \dot{\tilde{\mathrm{b}}}_{2}+\tilde{\mathrm{c}}_{2} \dot{\tilde{\mathrm{c}}}_{2}
\end{aligned}
$$




$$
\begin{aligned}
& =s\left[D_{t}^{1-q_{1}}\left(D_{t}^{q_{1}-1}\left[-\tilde{a}_{2}\left(y_{2}-x_{2}\right)-\tilde{a}_{1}\left(y_{1}-x_{1}\right)-\left(D_{t}^{p_{1}-1} e_{1}(t)\right) \frac{(t)^{-\left(q_{1}-1\right)-1}}{\Gamma\left(-\left(q_{1}-1\right)\right)}-\frac{5 s}{|s|+0.01}\right]\right)\right. \\
& \left.+\left(D_{t}^{\mathrm{q}_{1}-1} \mathrm{e}_{1}(\mathrm{t})\right) \frac{(\mathrm{t})^{-\left(\mathrm{q}_{1}-1\right)-1}}{\Gamma\left(-\left(\mathrm{q}_{1}-1\right)\right)}\right]+\mathrm{s}\left[\mathrm { D } _ { \mathrm { t } } ^ { 1 - \mathrm { q } _ { 2 } } \left(D _ { \mathrm { t } } ^ { \mathrm { q } _ { 2 } - 1 } \left[-\left(\tilde{\mathrm{b}}_{2}-\tilde{\mathrm{a}}_{2}\right) \mathrm{x}_{2}-\tilde{\mathrm{b}}_{2} \mathrm{y}_{2}-\tilde{\mathrm{b}}_{1} \mathrm{x}_{1}-\left(\mathrm{D}_{\mathrm{t}}^{\mathrm{q}_{2}-1} \mathrm{e}_{2}(\mathrm{t})\right)\right.\right.\right. \\
& \left.\left.\left.\frac{(\mathrm{t})^{-\left(\mathrm{q}_{2}-1\right)-1}}{\Gamma\left(-\left(\mathrm{q}_{2}-1\right)\right)}\right]\right)+\left(D_{\mathrm{t}}^{\mathrm{q}_{2}-1} \mathrm{e}_{2}(\mathrm{t})\right) \frac{(\mathrm{t})^{-\left(\mathrm{q}_{2}-1\right)-1}}{\Gamma\left(-\left(\mathrm{q}_{2}-1\right)\right)}-\frac{10 \mathrm{~s}}{|\mathrm{~s}|+0.01}\right]-\mathrm{s}\left[\mathrm { D } _ { \mathrm { t } } ^ { 1 - \mathrm { q } _ { 3 } } \left(D _ { \mathrm { t } } ^ { 1 - \mathrm { q } _ { 3 } } \left[\tilde{\mathrm{c}}_{2} \mathrm{z}_{2}+\tilde{\mathrm{c}}_{1} \mathrm{z}_{1}\right.\right.\right. \\
& \left.\left.\left.-\left(D_{\mathrm{t}}^{\mathrm{q}_{3}-1} \mathrm{e}_{3}(\mathrm{t})\right) \frac{(\mathrm{t})^{-\left(\mathrm{q}_{3}-1\right)-1}}{\Gamma\left(-\left(\mathrm{q}_{3}-1\right)\right)}\right]\right)+\left(\mathrm{D}_{\mathrm{t}}^{\mathrm{q}_{3}-1} \mathrm{e}_{3}(\mathrm{t})\right) \frac{(\mathrm{t})^{-\left(\mathrm{q}_{3}-1\right)-1}}{\Gamma\left(-\left(\mathrm{q}_{3}-1\right)\right)}\right]+\tilde{\mathrm{a}}_{1} \dot{\tilde{\mathrm{a}}}_{1}+\tilde{\mathrm{b}}_{1} \dot{\tilde{\mathrm{b}}}_{1}+\tilde{\mathrm{c}}_{1} \dot{\tilde{\mathrm{c}}}_{1}+\tilde{\mathrm{a}}_{2} \dot{\tilde{\mathrm{a}}}_{2} \\
& +\tilde{\mathrm{b}}_{2} \dot{\tilde{\mathrm{b}}}_{2}+\tilde{\mathrm{c}}_{2} \dot{\tilde{\mathrm{c}}}_{2} \text {. }
\end{aligned}
$$

Since $\forall \mathrm{q} \in[0,1]$, we have $(1-\mathrm{q})>0$ and $(\mathrm{q}-1)<0$. Now, using (5) and introducing update laws (29) in (32) one obtains

$$
\begin{aligned}
\dot{V}= & s\left(-\tilde{\mathrm{a}}_{2}\left(\mathrm{y}_{2}-\mathrm{x}_{2}\right)-\tilde{\mathrm{a}}_{1}\left(\mathrm{y}_{1}-\mathrm{x}_{1}\right)-\frac{5 \mathrm{~s}}{|\mathrm{~s}|+0.01}\right)+\mathrm{s}\left(-\left(\tilde{\mathrm{b}}_{2}-\tilde{\mathrm{a}}_{2}\right) \mathrm{x}_{2}-\tilde{\mathrm{b}}_{2} \mathrm{y}_{2}-\tilde{\mathrm{b}}_{1} \mathrm{x}_{1}\right. \\
& \left.-\frac{10 \mathrm{~s}}{|\mathrm{~s}|+0.01}\right)-\mathrm{s}\left(\tilde{\mathrm{c}}_{2} \mathrm{z}_{2}+\tilde{\mathrm{c}}_{1} \mathrm{z}_{1}\right)+\tilde{\mathrm{a}}_{1}\left(\mathrm{~s}\left(\mathrm{y}_{1}-\mathrm{x}_{1}\right)\right)+\tilde{\mathrm{b}}_{1}\left(\mathrm{sx}_{1}\right)+\tilde{\mathrm{c}}_{1}\left(\mathrm{sz}_{1}\right) \\
& +\tilde{\mathrm{a}}_{2}\left(\mathrm{~s}\left(\mathrm{y}_{2}-\mathrm{x}_{2}\right)-\mathrm{sx}_{2}\right)+\tilde{\mathrm{b}}_{2}\left(\mathrm{~s}\left(\mathrm{x}_{2}+\mathrm{y}_{2}\right)\right)+\tilde{\mathrm{c}}_{2}\left(\mathrm{sz}_{2}\right) .
\end{aligned}
$$

Then, (33) reduces to

$$
\dot{\mathrm{V}}=-\frac{15 \mathrm{~s}^{2}}{|\mathrm{~s}|+0.01} \text {. }
$$

Since $\mathrm{s}^{2}>0$ and $|\mathrm{s}|>0$ both hold true, then, when $\mathrm{e} \neq 0$ and $\mathrm{ck}>0$, the inequality $\dot{\mathrm{V}}<0$ holds. According to the Lyapunov stability theory [25] V is positive-definite, and $\dot{V}$ is negative-definite. Thus, the trajectories of the fractional error dynamical system (26) asymptotically converge to $s(t)=0$. Therefore, the state variables of the of the drive system (24) and the states variables of the response (25) system can be anti-synchronized asymptotically and globally with the control law (28) and the adaptive parameter update laws (29). Here, the proof is completed.

\subsection{Numerical simulations}

Numerical simulations are presented to verify the effectiveness of the proposed adaptive sliding mode antisynchronization between the fractional-order Lorenz and Chen systems using Adams-Bashforth-Moulton method. The parameters are chosen to be $a_{1}=10, b_{1}=28, c_{1}=8 / 3, a_{2}=35, b_{2}=28$, and $c_{2}=3$. The initial conditions of the drive system (24) and response system (25) are set to $\mathrm{x}_{1}(0)=6, \mathrm{y}_{1}(0)=3, \mathrm{z}_{1}(0)=$ $7, \mathrm{x}_{2}(0)=2, \mathrm{y}_{2}(0)=7$, and $\mathrm{z}_{2}(0)=4$. Moreover, the initial values of the unknown parameters are chosen as $\tilde{\mathrm{a}}_{1}(0)=10, \tilde{\mathrm{b}}_{1}(0)=10, \tilde{\mathrm{c}}_{1}(0)=10, \tilde{\mathrm{a}}_{2}(0)=10, \tilde{\mathrm{b}}_{2}(0)=10$, and $\tilde{\mathrm{c}}_{2}(0)=10$. The simulation results are shown in Figs. (1)-(2). Figs. (1) (a)-(c) depicts the time response of the drive (24) and response (25) systems, while Fig. (2) (a) depicts the time response of the error states $e_{1}, e_{2}$, and $e_{3}$ under the control law (28) and the adaptive parameter update laws (29). Figs. (2) (b)-(c) depicts the temporal response of the unknown parameters $\tilde{\mathrm{a}}_{1}, \tilde{\mathrm{b}}_{1}, \tilde{\mathrm{c}}_{1}, \tilde{\mathrm{a}}_{2}, \tilde{\mathrm{b}}_{2}$, and $\tilde{\mathrm{c}}_{2}$ of the drive (24) and response (25) systems. 
(a)
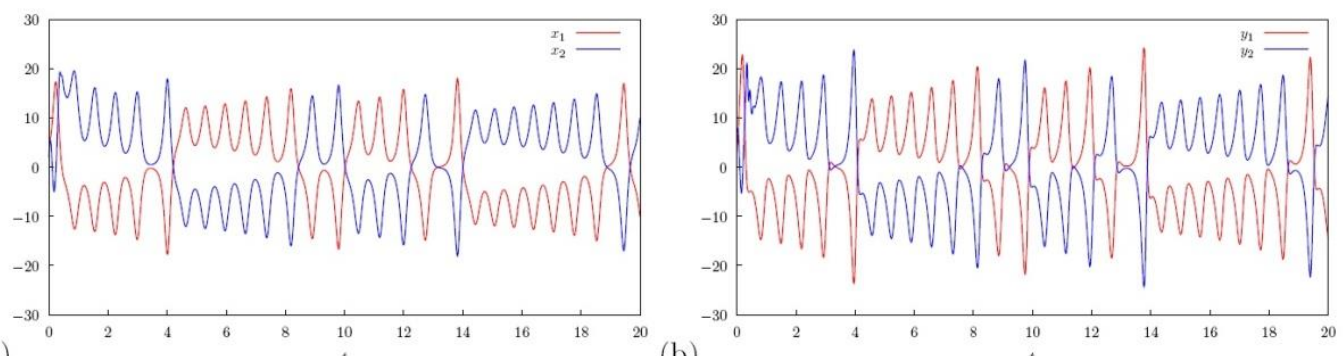

(b)

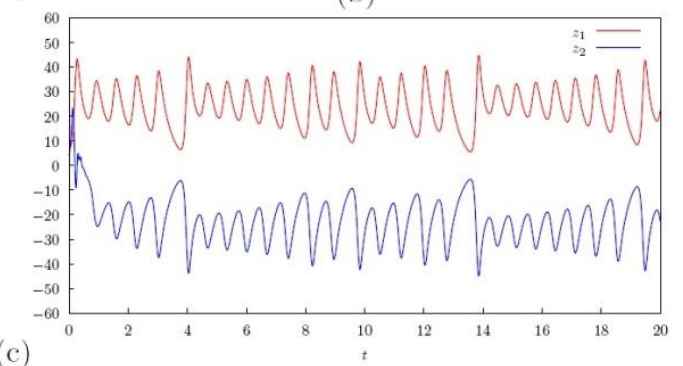

(c)

Figure 1: Anti-synchronization of the drive (24) and the response systems (25). The plotted signals are (a) $x_{1}$ and $x_{2}$, (b) $y_{1}$ and $y_{2}$, and (c) $z_{1}$ and $z_{2}$.

(a)
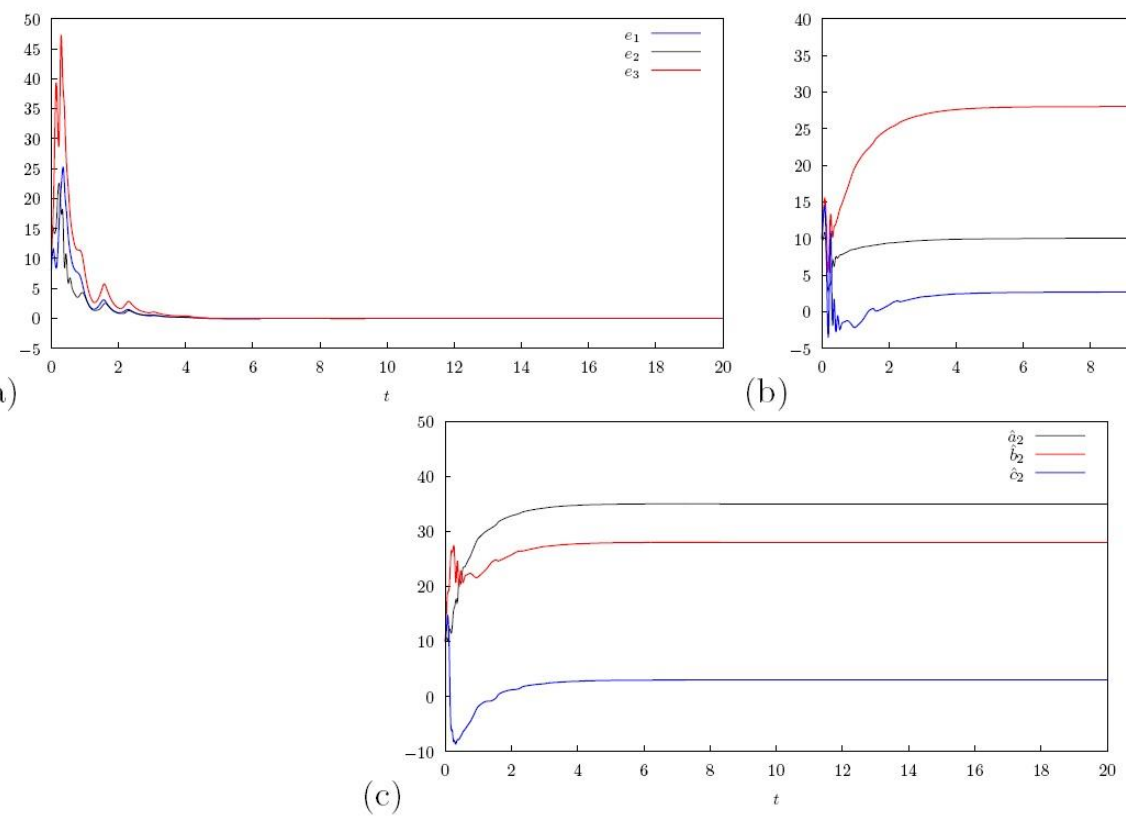

Figure 2: (a) Anti-synchronization error signals $e_{1}, e_{2}$, and $e_{3}$ between the drive (24) and response systems (25) under the controller (28). (b) Parameter estimates of the drive system (24). (c) Parameter estimates of the response system (25).

5. Modified adaptive sliding mode anti-synchronization of two fractional order hyperchaotic systems

This section investigates the anti-synchronisation behaviour between two different fractional-order hyperchaotic systems using the modified adaptive sliding-mode control method. The drive system is assumed to be a fractional-order hyperchaotic Lorenz system ( $\mathrm{Li}$, Wang \& Yang, 2014), while a fractional-order hyperchaotic Lü system (Li, Wang \& Yang,2014), is taken as the response. The definitions of both systems have unknown parameters:

$$
\begin{aligned}
& \mathrm{D}_{\mathrm{t}}^{\mathrm{q}_{1}} \mathrm{x}_{1}=\mathrm{a}_{1}\left(\mathrm{y}_{1}-\mathrm{x}_{1}\right)+\mathrm{w}_{1} \text {, } \\
& \mathrm{D}_{\mathrm{t}}^{\mathrm{q}_{2}} \mathrm{y}_{1}=\mathrm{b}_{1} \mathrm{x}_{1}-\mathrm{x}_{1} \mathrm{z}_{1}-\mathrm{y}_{1} \text {, }
\end{aligned}
$$




$$
\begin{aligned}
& D_{t}^{q_{3}} z_{1}=x_{1} y_{1}-c_{1} z_{1}, \\
& D_{t}^{q_{4}} w_{1}=-y_{1} z_{1}+d_{1} w_{1},
\end{aligned}
$$

and

$$
\begin{aligned}
& D_{t}^{q_{1}} x_{2}=a_{2}\left(y_{2}-x_{2}\right)+w_{2}+u_{1}, \\
& D_{t}^{q_{2}} y_{2}=b_{2} y_{2}-x_{2} z_{2}+u_{2}, \\
& D_{t}^{q_{3}} z_{2}=x_{2} y_{2}-c_{2} z_{2}+u_{3}, \\
& D_{t}^{q_{4}} w_{2}=x_{2} z_{2}+d_{2} w_{2}+u_{4},
\end{aligned}
$$

where the variables $\left(\mathrm{u}_{1}, \mathrm{u}_{2}, \mathrm{u}_{3}, \mathrm{u}_{4}\right)^{\mathrm{T}}$ are controllers to be designed. Let $\mathrm{e}_{1}=\mathrm{x}_{2}+\mathrm{x}_{1}, \mathrm{e}_{2}=\mathrm{y}_{2}+\mathrm{y}_{1}, \mathrm{e}_{3}=\mathrm{z}_{2}+\mathrm{z}_{1}$ and $e_{4}=w_{2}+w_{1}$. Then, we get the following error dynamic system between the drive (35) and response (36) systems

$$
\begin{aligned}
& D_{t}^{q_{1}} e_{1}(t)=a_{2}\left(y_{2}-x_{2}\right)+a_{1}\left(y_{1}-x_{1}\right)+e_{4}+u_{1}, \\
& D_{t}^{q_{2}} e_{2}(t)=b_{2} y_{2}-x_{2} z_{2}+b_{1} x_{1}-x_{1} z_{1}-y_{1}+u_{2}, \\
& D_{t}^{q_{3}} e_{3}(t)=x_{2} y_{2}-c_{2} z_{2}+x_{1} y_{1}-c_{1} z_{1}+u_{3}, \\
& D_{t}^{q_{4}} e_{4}(t)=x_{1} z_{1}+d_{2} w_{2}-y_{1} z_{1}+d_{1} w_{1}+u_{4} .
\end{aligned}
$$

The goal of the modified adaptive sliding-mode control is to find an effective controller function $\left(\mathrm{u}_{1}, \mathrm{u}_{2}, \mathrm{u}_{3}, \mathrm{u}_{4}\right)^{\mathrm{T}}$ capable anti-synchronizing the states of the response and drive systems with a parameter estimation update law. An appropriate sliding surface can be chosen as

$$
\begin{aligned}
& s(e)=e_{1}+e_{2}+3 e_{3}-3 e_{4}, \\
& w(t)=\frac{s}{|s|+0.01},
\end{aligned}
$$

It is assumed that the constant vectors are $\mathrm{c}=(1,1,3,-3), \mathrm{k}=(0,10,0,0)^{\mathrm{T}}$, and $\gamma=0.01$. The adaptive slidingmode controller of the error dynamic system (37) can be calculated as follows

$$
\begin{aligned}
& \mathrm{u}_{1}=-\mathrm{a}_{2}\left(\mathrm{y}_{2}-\mathrm{x}_{2}\right)-\mathrm{a}_{1}\left(\mathrm{y}_{1}-\mathrm{x}_{1}\right)-\mathrm{e}_{4}+\mathrm{D}_{\mathrm{t}}^{\mathrm{q}_{1}-1}\left[-\hat{\mathrm{a}}_{2}\left(\mathrm{y}_{2}-\mathrm{x}_{2}\right)-\hat{\mathrm{a}}_{1}\left(\mathrm{y}_{1}-\mathrm{x}_{1}\right)\right. \\
& \left.-\left(\mathrm{D}_{\mathrm{t}}^{\mathrm{q}_{1}-1} \mathrm{e}_{1}(\mathrm{t})\right) \frac{(\mathrm{t})^{-\left(\mathrm{q}_{1}-1\right)-1}}{\Gamma\left(-\left(\mathrm{q}_{1}-1\right)\right)}\right] \\
& \mathrm{u}_{2}=-\mathrm{b}_{2} \mathrm{y}_{2}+\mathrm{x}_{2} \mathrm{z}_{2}-\mathrm{b}_{1} \mathrm{x}_{1}+\mathrm{x}_{1} \mathrm{z}_{1}-\mathrm{y}_{1}+\mathrm{D}_{\mathrm{t}}^{\mathrm{q}_{2}-1}\left[-\hat{\mathrm{b}}_{2} \mathrm{y}_{2}-\hat{\mathrm{b}}_{1} \mathrm{x}_{1}-\left(\mathrm{D}_{\mathrm{t}}^{\mathrm{q}_{2}-1} \mathrm{e}_{2}(\mathrm{t})\right)\right. \\
& \left.\frac{(\mathrm{t})^{-\left(\mathrm{q}_{2}-1\right)-1}}{\Gamma\left(-\left(\mathrm{q}_{2}-1\right)\right)}-\frac{10 \mathrm{~s}}{|\mathrm{~s}|+0.01}\right], \\
& \mathrm{u}_{3}=-\mathrm{x}_{2} \mathrm{y}_{2}+\mathrm{c}_{2} \mathrm{z}_{2}-\mathrm{x}_{1} \mathrm{y}_{1}+\mathrm{c}_{1} \mathrm{z}_{1}+\mathrm{D}_{\mathrm{t}}^{\mathrm{p}_{3}-1}\left[\hat{\mathrm{c}}_{2} \mathrm{z}_{2}+\widehat{\mathrm{c}}_{1} \mathrm{z}_{1}-\left(\mathrm{D}_{\mathrm{t}}^{\mathrm{q}_{3}-1} \mathrm{e}_{3}(\mathrm{t})\right) \frac{(\mathrm{t})^{-\left(\mathrm{q}_{3}-1\right)-1}}{\Gamma\left(-\left(\mathrm{q}_{3}-1\right)\right)}\right], \\
& \mathrm{u}_{4}=-\mathrm{x}_{1} \mathrm{z}_{1}-\mathrm{d}_{2} \mathrm{w}_{2}+\mathrm{y}_{1} \mathrm{z}_{1}-\mathrm{d}_{1} \mathrm{w}_{1}+\mathrm{D}_{\mathrm{t}}^{\mathrm{q}_{4}-1}\left[-\hat{\mathrm{d}}_{2} \mathrm{w}_{2}-\hat{\mathrm{d}}_{1} \mathrm{w}_{1}-\left(\mathrm{D}_{\mathrm{t}}^{\mathrm{q}_{4}-1} \mathrm{e}_{4}(\mathrm{t})\right) \frac{(\mathrm{t})^{-\left(\mathrm{q}_{4}-1\right)-1}}{\Gamma\left(-\left(\mathrm{q}_{4}-1\right)\right)}\right] .
\end{aligned}
$$

The adaptive laws for estimating the parameters $\hat{a}_{1}, \hat{b}_{1}, \hat{c}_{1}, \hat{\mathrm{d}}_{1}, \hat{\mathrm{a}}_{2}, \hat{\mathrm{b}}_{2}, \hat{\mathrm{c}}_{2}$ and $\hat{\mathrm{d}}_{2}$ are chosen as follows:

$$
\begin{aligned}
& \dot{\tilde{\mathrm{a}}}_{1}=\mathrm{s}\left(\mathrm{y}_{1}-\mathrm{x}_{1}\right) \\
& \dot{\tilde{\mathrm{b}}}_{1}=\mathrm{sx}_{1}, \\
& \dot{\tilde{\mathrm{c}}}_{1}=-3 \mathrm{sz}_{1}, \\
& \dot{\tilde{\mathrm{d}}}_{1}=-3 \mathrm{sw}_{1}, \\
& \dot{\tilde{\mathrm{a}}}_{2}=\mathrm{s}\left(\mathrm{y}_{2}-\mathrm{x}_{2}\right), \\
& \dot{\tilde{\mathrm{b}}}_{2}=\mathrm{sy}_{2},
\end{aligned}
$$




$$
\begin{aligned}
& \dot{\tilde{\mathrm{c}}}_{2}=-3 \mathrm{sz}_{2}, \\
& \dot{\hat{\mathrm{d}}}_{2}=-3 \mathrm{sw}_{2},
\end{aligned}
$$

Theorem 3. The state variables of the of the drive system (35) and the states variables of the response (36) system can be anti-synchronized asymptotically and globally for all initial conditions using the control law (39) and the adaptive parameter update laws (40).

Proof. Substituting (39) into (37), this yields

$$
\begin{aligned}
& D_{t}^{\mathrm{q}_{1}} \mathrm{e}_{1}(\mathrm{t})=\mathrm{D}_{\mathrm{t}}^{\mathrm{q}_{1}-1}\left[-\hat{\mathrm{a}}_{2}\left(\mathrm{y}_{2}-\mathrm{x}_{2}\right)-\hat{\mathrm{a}}_{1}\left(\mathrm{y}_{1}-\mathrm{x}_{1}\right)-\left(\mathrm{D}_{\mathrm{t}}^{\mathrm{q}_{1}-1} \mathrm{e}_{1}(\mathrm{t})\right) \frac{(\mathrm{t})^{-\left(\mathrm{q}_{1}-1\right)-1}}{\Gamma\left(-\left(\mathrm{q}_{1}-1\right)\right)}\right], \\
& \mathrm{D}_{\mathrm{t}}^{\mathrm{q}_{2}} \mathrm{e}_{2}(\mathrm{t})=D_{\mathrm{t}}^{\mathrm{q}_{2}-1}\left[-\tilde{\mathrm{b}}_{2} \mathrm{y}_{2}-\tilde{\mathrm{b}}_{1} \mathrm{x}_{1}-\left(\mathrm{D}_{\mathrm{t}}^{\mathrm{q}_{2}-1} \mathrm{e}_{2}(\mathrm{t})\right) \frac{(\mathrm{t})^{-\left(\mathrm{q}_{2}-1\right)-1}}{\Gamma\left(-\left(\mathrm{q}_{2}-1\right)\right)}-\frac{10 \mathrm{~s}}{|\mathrm{~s}|+0.01}\right], \\
& D_{\mathrm{t}}^{\mathrm{q}_{3}} \mathrm{e}_{3}(\mathrm{t})=D_{\mathrm{t}}^{\mathrm{q}_{3}-1}\left[\tilde{\mathrm{c}}_{2} \mathrm{z}_{2}+\tilde{\mathrm{c}}_{1} \mathrm{z}_{1}-\left(\mathrm{D}_{\mathrm{t}}^{\mathrm{q}_{3}-1} \mathrm{e}_{3}(\mathrm{t})\right) \frac{(\mathrm{t})^{-\left(\mathrm{q}_{3}-1\right)-1}}{\Gamma\left(-\left(\mathrm{q}_{3}-1\right)\right)}\right], \\
& D_{\mathrm{t}}^{\mathrm{q}_{4}} \mathrm{e}_{4}(\mathrm{t})=D_{\mathrm{t}}^{\mathrm{q}_{4}-1}\left[-\tilde{\mathrm{d}}_{2} \mathrm{w}_{2}-\tilde{\mathrm{d}}_{1} \mathrm{w}_{1}-\left(D_{\mathrm{t}}^{\mathrm{q}_{4}-1} \mathrm{e}_{4}(\mathrm{t})\right) \frac{(\mathrm{t})^{-\left(\mathrm{q}_{4}-1\right)-1}}{\Gamma\left(-\left(\mathrm{q}_{4}-1\right)\right)}\right],
\end{aligned}
$$

where $\tilde{\mathrm{a}}_{1}=\hat{\mathrm{a}}_{1}-\mathrm{a}_{1}, \tilde{\mathrm{b}}_{1}=\hat{\mathrm{b}}_{1}-\mathrm{b}_{1}, \tilde{\mathrm{c}}_{1}=\hat{\mathrm{c}}_{1}-\mathrm{c}_{1}, \tilde{\mathrm{d}}_{1}=\widehat{\mathrm{d}}_{1}-\mathrm{d}_{1}, \tilde{\mathrm{a}}_{2}=\hat{\mathrm{a}}_{2}-\mathrm{a}_{2}, \tilde{\mathrm{b}}_{2}=\hat{\mathrm{b}}_{2}-\mathrm{b}_{2}, \tilde{\mathrm{c}}_{2}=\hat{\mathrm{c}}_{2}-\mathrm{c}_{2}$, and $\tilde{\mathrm{d}}_{2}=\hat{\mathrm{d}}_{2}-\mathrm{d}_{2}$. Selecting a Lyapunov function candidate in the form of

$$
\mathrm{V}=\frac{1}{2}\left(\mathrm{~s}^{2}+\tilde{\mathrm{a}}_{1}^{2}+\tilde{\mathrm{b}}_{1}^{2}+\tilde{\mathrm{c}}_{1}^{2}+\tilde{\mathrm{d}}_{1}^{2}+\tilde{\mathrm{a}}_{2}^{2}+\tilde{\mathrm{b}}_{2}^{2}+\tilde{\mathrm{c}}_{2}^{2}+\tilde{\mathrm{d}}_{2}^{2}\right) \text {. }
$$

Taking the derivative of (42) with respect to time using (4), one has

$$
\begin{aligned}
& \dot{\mathrm{V}}=\left(\mathrm{s} \dot{\mathrm{s}}+\tilde{\mathrm{a}}_{1} \dot{\tilde{\mathrm{a}}}_{1}+\tilde{\mathrm{b}}_{1} \dot{\tilde{\mathrm{b}}}_{1}+\tilde{\mathrm{c}}_{1} \dot{\tilde{\mathrm{c}}}_{1}+\tilde{\mathrm{d}}_{1} \dot{\tilde{\mathrm{d}}}_{1}+\tilde{\mathrm{a}}_{2} \dot{\tilde{\mathrm{a}}}_{2}+\tilde{\mathrm{b}}_{2} \dot{\mathrm{b}}_{2}+\tilde{\mathrm{c}}_{2} \dot{\tilde{\mathrm{c}}}_{2}+\tilde{\mathrm{d}}_{2} \dot{\tilde{\mathrm{d}}}_{2}\right) \\
& =s\left[D_{t}^{1-q_{1}}\left(D_{t}^{q_{1}} e_{1}(t)\right)+\left(D_{t}^{q_{1}-1} e_{1}(t)\right) \frac{(t-)^{-\left(q_{1}-1\right)-1}}{\Gamma\left(-\left(q_{1}-1\right)\right)}\right]+s\left[D_{t}^{1-q_{2}}\left(D_{t}^{q_{2}} e_{2}(t)\right)+\left(D_{t}^{q_{2}-1} e_{2}(t)\right)\right. \\
& \left.\frac{(\mathrm{t})^{-\left(\mathrm{q}_{2}-1\right)-1}}{\Gamma\left(-\left(\mathrm{q}_{2}-1\right)\right)}\right]+3 \mathrm{~s}\left[\mathrm{D}_{\mathrm{t}}^{1-\mathrm{q}_{3}}\left(D_{\mathrm{t}}^{\mathrm{q}_{3}} \mathrm{e}_{3}(\mathrm{t})\right)+\left(\mathrm{D}_{\mathrm{t}}^{\mathrm{q}_{3}-1} \mathrm{e}_{3}(\mathrm{t})\right) \frac{(\mathrm{t})^{-\left(\mathrm{q}_{3}-1\right)-1}}{\Gamma\left(-\left(\mathrm{q}_{3}-1\right)\right)}\right]-3 \mathrm{~s}\left[\mathrm{D}_{\mathrm{t}}^{1-\mathrm{q}_{4}}\left(\mathrm{D}_{\mathrm{t}}^{\mathrm{q}_{4}} \mathrm{e}_{4}(\mathrm{t})\right)\right. \\
& \left.+\left(D_{\mathrm{t}}^{\mathrm{p}_{4}-1} \mathrm{e}_{4}(\mathrm{t})\right) \frac{(\mathrm{t})^{-\left(\mathrm{p}_{4}-1\right)-1}}{\Gamma\left(-\left(\mathrm{p}_{4}-1\right)\right)}\right]+\widetilde{\mathrm{a}}_{1} \dot{\tilde{\mathrm{a}}}_{1}+\tilde{\mathrm{b}}_{1} \dot{\tilde{\mathrm{b}}}_{1}+\tilde{\mathrm{c}}_{1} \dot{\tilde{\mathrm{c}}}_{1}+\tilde{\mathrm{d}}_{1} \dot{\tilde{\mathrm{d}}}_{1}+\tilde{\mathrm{a}}_{2} \dot{\tilde{\mathrm{a}}}_{2}+\tilde{\mathrm{b}}_{2} \dot{\tilde{\mathrm{b}}}_{2}+\tilde{\mathrm{c}}_{2} \dot{\tilde{\mathrm{c}}}_{2}+\tilde{\mathrm{d}}_{2} \dot{\tilde{\mathrm{d}}}_{2} . \\
& =s\left[D_{t}^{1-q_{1}}\left(D_{t}^{q_{1}-1}\left[-\tilde{a}_{2}\left(y_{2}-x_{2}\right)-\tilde{a}_{1}\left(y_{1}-x_{1}\right)-\left(D_{t}^{q_{1}-1} e_{1}(t)\right) \frac{(t)-\left(q_{1}-1\right)-1}{\Gamma\left(-\left(q_{1}-1\right)\right)}\right]\right)\right. \\
& \left.+\left(D_{t}^{q_{1}-1} e_{1}(t)\right) \frac{\left(t^{-\left(q_{1}-1\right)-1}\right.}{\Gamma\left(-\left(\mathrm{q}_{1}-1\right)\right)}\right]+s\left[D _ { t } ^ { 1 - q _ { 2 } } \left(D _ { t } ^ { q _ { 2 } - 1 } \left[-\tilde{b}_{2} y_{2}-\tilde{b}_{1} x_{1}-\left(D_{t}^{q_{2}-1} e_{2}(t)\right)\right.\right.\right. \\
& \left.\left.\left.\frac{(\mathrm{t})^{-\left(\mathrm{q}_{2}-1\right)-1}}{\Gamma\left(-\left(\mathrm{q}_{2}-1\right)\right)}\right]\right)+\left(D_{\mathrm{t}}^{\mathrm{q}_{2}-1} \mathrm{e}_{2}(\mathrm{t})\right) \frac{(\mathrm{t})^{-\left(\mathrm{q}_{2}-1\right)-1}}{\Gamma\left(-\left(\mathrm{q}_{2}-1\right)\right)}-\frac{10 \mathrm{~s}}{|\mathrm{~s}|+0.01}\right]+3 \mathrm{~s}\left[\mathrm { D } _ { \mathrm { t } } ^ { 1 - \mathrm { q } _ { 3 } } \left(\mathrm { D } _ { \mathrm { t } } ^ { 1 - \mathrm { q } _ { 3 } } \left[\tilde{\mathrm{c}}_{2} \mathrm{z}_{2}+\tilde{\mathrm{c}}_{1} \mathrm{z}_{1}\right.\right.\right. \\
& \left.\left.\left.-\left(D_{\mathrm{t}}^{\mathrm{q}_{3}-1} \mathrm{e}_{3}(\mathrm{t})\right) \frac{(\mathrm{t})^{-\left(\mathrm{q}_{3}-1\right)-1}}{\Gamma\left(-\left(\mathrm{q}_{3}-1\right)\right)}\right]\right)+\left(D_{\mathrm{t}}^{\mathrm{q}_{3}-1} \mathrm{e}_{3}(\mathrm{t})\right) \frac{(\mathrm{t})^{-\left(\mathrm{q}_{3}-1\right)-1}}{\Gamma\left(-\left(\mathrm{q}_{3}-1\right)\right)}\right]-3 \mathrm{~s}\left[\mathrm { D } _ { \mathrm { t } } ^ { 1 - \mathrm { q } _ { 4 } } \left(D _ { \mathrm { t } } ^ { 1 - \mathrm { q } _ { 4 } } \left[-\tilde{\mathrm{d}}_{2} \mathrm{w}_{2}-\tilde{\mathrm{d}}_{1} \mathrm{w}_{1}\right.\right.\right. \\
& \left.\left.\left.-\left(\mathrm{D}_{\mathrm{t}}^{\mathrm{q}_{4}-1} \mathrm{e}_{4}(\mathrm{t})\right) \frac{(\mathrm{t})^{-\left(\mathrm{q}_{4}-1\right)-1}}{\Gamma\left(-\left(\mathrm{q}_{4}-1\right)\right)}\right]\right)+\left(\mathrm{D}_{\mathrm{t}}^{\mathrm{q}_{4}-1} \mathrm{e}_{4}(\mathrm{t})\right) \frac{(\mathrm{t})^{-\left(\mathrm{q}_{4}-1\right)-1}}{\Gamma\left(-\left(\mathrm{q}_{4}-1\right)\right)}\right]+\tilde{\mathrm{a}}_{1} \dot{\tilde{\mathrm{a}}}_{1}+\tilde{\mathrm{b}}_{1} \dot{\tilde{\mathrm{b}}}_{1}+\tilde{\mathrm{c}}_{1} \dot{\tilde{\mathrm{c}}}_{1}+\tilde{\mathrm{d}}_{1} \dot{\tilde{\mathrm{d}}}_{1}+\tilde{\mathrm{a}}_{2} \dot{\tilde{\mathrm{a}}}_{2} \\
& +\widetilde{\mathrm{b}}_{2} \dot{\tilde{\mathrm{b}}}_{2}+\tilde{\mathrm{c}}_{2} \dot{\tilde{\mathrm{c}}}_{2}+\tilde{\mathrm{d}}_{2} \dot{\tilde{\mathrm{d}}}_{2} \text {. }
\end{aligned}
$$

Since $\forall \mathrm{q} \in[0,1]$, we have $(1-\mathrm{q})>0$ and $(\mathrm{q}-1)<0$. Now, using (5) and introducing update laws (40) in (43) one obtains

$$
\begin{aligned}
\dot{V}= & s\left(-\tilde{\mathrm{a}}_{2}\left(\mathrm{y}_{2}-\mathrm{x}_{2}\right)-\tilde{\mathrm{a}}_{1}\left(\mathrm{y}_{1}-\mathrm{x}_{1}\right)\right)+\mathrm{s}\left(-\tilde{\mathrm{b}}_{2} \mathrm{y}_{2}-\tilde{\mathrm{b}}_{1} \mathrm{x}_{1}-\frac{10 \mathrm{~s}}{|\mathrm{~s}|+0.01}\right)+3 \mathrm{~s}\left(\tilde{\mathrm{c}}_{2} \mathrm{z}_{2}+\tilde{\mathrm{c}}_{1} \mathrm{z}_{1}\right) \\
& -3 \mathrm{~s}\left(-\tilde{\mathrm{d}}_{2} \mathrm{w}_{2}-\tilde{\mathrm{d}}_{1} \mathrm{w}_{1}\right)+\tilde{\mathrm{a}}_{1}\left(\mathrm{~s}\left(\mathrm{y}_{1}-\mathrm{x}_{1}\right)\right)+\tilde{\mathrm{b}}_{1}\left(\mathrm{sx}_{1}\right)+\tilde{\mathrm{c}}_{1}\left(-3 s \mathrm{z}_{1}\right)+\tilde{\mathrm{d}}_{1}\left(-3 \mathrm{sw}_{1}\right) \\
& +\tilde{\mathrm{a}}_{2}\left(\left(\mathrm{y}_{2}-\mathrm{x}_{2}\right)\right)+\tilde{\mathrm{b}}_{2}\left(\mathrm{sy}_{2}\right)+\tilde{\mathrm{c}}_{2}\left(-3 \mathrm{sz}_{2}\right)+\tilde{\mathrm{d}}_{2}\left(-3 s \mathrm{sw}_{2}\right) .
\end{aligned}
$$

Then, (33) reduces to 


$$
\dot{\mathrm{V}}=-\frac{10 \mathrm{~s}^{2}}{|\mathrm{~s}|+0.01} .
$$

Since $s^{2}>0$ and $|s|>0$ both hold true, then, when $\mathrm{e} \neq 0$ and ck $>0$, the inequality $\dot{V}<0$ holds. According to the Lyapunov stability theory [25] V is positive-definite, and $\dot{V}$ is negative-definite. Thus, the trajectories of the fractional error dynamical system (26) asymptotically converge to $s(t)=0$. Therefore, the state variables of the of the drive system (35) and the states variables of the response (36) system can be anti-synchronized asymptotically and globally with the control law (39) and the adaptive parameter update laws (40). Here, the proof is completed.

\subsection{Numerical simulations}

Numerical simulations are presented to verify the effectiveness of the proposed adaptive sliding mode antisynchronization between the fractional-order hyperchaotic Lorenz system and the fractional-order hyperchaotic Lü systemusing Adams-Bashforth-Moulton method. The parameters are chosen to be $\mathrm{a}_{1}=10, \mathrm{~b}_{1}=28, \mathrm{c}_{1}=$ $8 / 3, d_{1}=-1, a_{2}=36, b_{2}=20, c_{2}=3$, and $d_{2}=1.3$. The initial conditions of the drive system (35) and response system $(36)$ are set to $\mathrm{x}_{1}(0)=6, \mathrm{y}_{1}(0)=3, \mathrm{z}_{1}(0)=7, \mathrm{w}_{1}(0)=2, \mathrm{x}_{2}(0)=2, \mathrm{y}_{2}(0)=7, \mathrm{z}_{2}(0)=4$ and $w_{2}(0)=4$. Moreover, the initial values of the unknown parameters are chosen as $\tilde{a}_{1}(0)=1, \tilde{b}_{1}(0)=1$, $\tilde{\mathrm{c}}_{1}(0)=1, \tilde{\mathrm{d}}_{1}(0)=1 \tilde{\mathrm{a}}_{2}(0)=10$, and $\tilde{\mathrm{b}}_{2}(0)=10, \tilde{\mathrm{c}}_{2}(0)=10, \tilde{\mathrm{d}}_{2}(0)=1$. The simulation results are shown in Figs (3)-(4). Figs. (3) (a)-(d) depicts the time response of the drive (35) and response (36) systems, while Fig. (4) (a) depicts the time response of the error states $e_{1}, e_{2}, e_{3}$, and $e_{4}$ under the control law (39) and the adaptive parameter update laws (40). Figs. (4) (b)-(c) depicts the temporal response of the unknown parameters $\tilde{\mathrm{a}}_{1}, \tilde{\mathrm{b}}_{1}, \tilde{\mathrm{c}}_{1}, \tilde{\mathrm{d}}_{1}, \tilde{\mathrm{a}}_{2}, \tilde{\mathrm{b}}_{2}, \tilde{\mathrm{c}}_{2}$, and $\tilde{\mathrm{d}}_{2}$ of the drive (35) and response (36) systems.
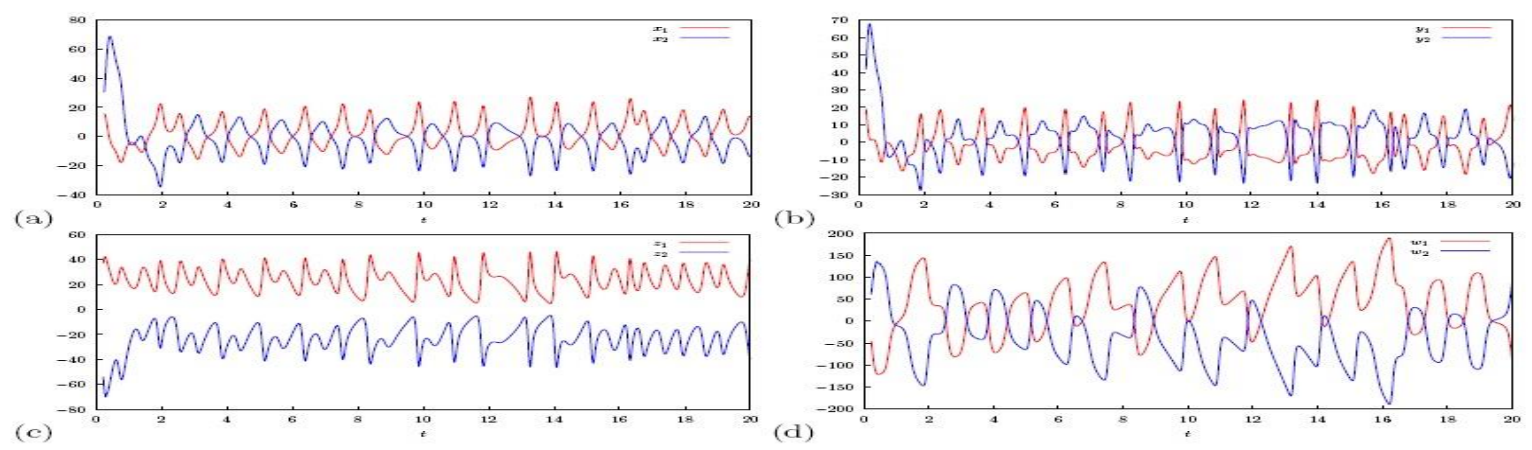

Figure 4: Anti-synchronization of the drive (35) and the response systems (36). The plotted signals are (a) $x_{1}$ and $x_{2}$, (b) $y_{1}$ and $y_{2}$, (c) $z_{1}$ and $z_{2}$ and (d) $w_{1}$ and $w_{2}$.
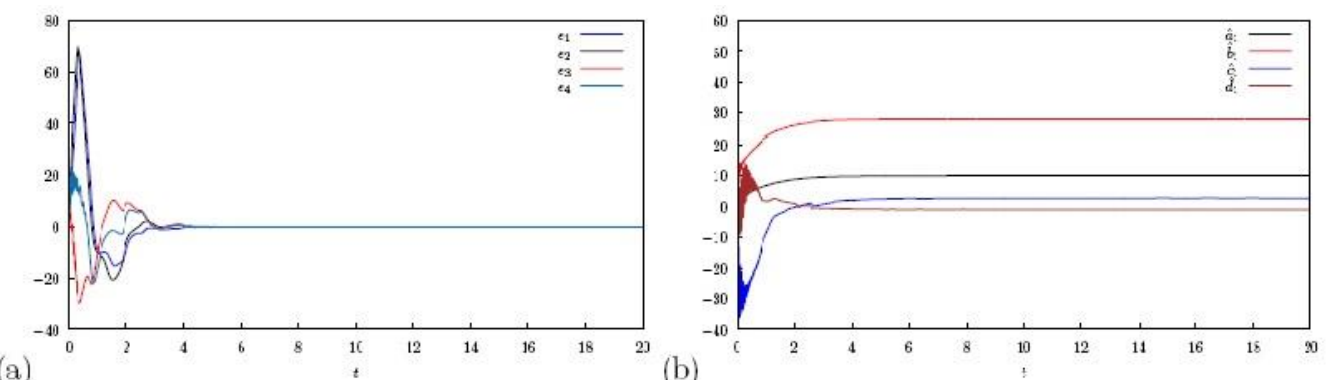

(a)

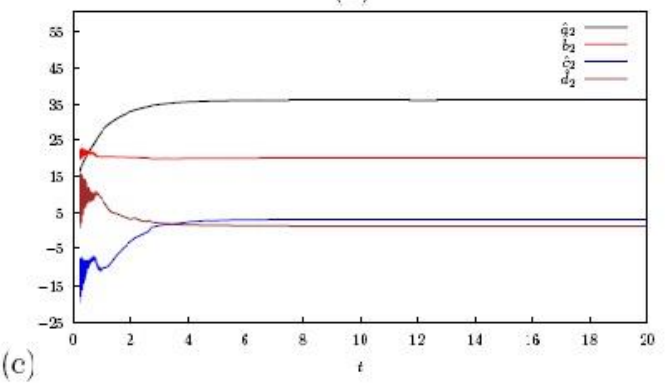


Figure 5: (a) Anti-synchronization error signals $e_{1}, e_{2}, e_{3}$, and $e_{4}$ between the drive (35) and response systems (36) under the controller (39). (b) Parameter estimates of the drive system (35). (c) Parameter estimates of the response system (36).

\section{Conclusion}

We presented a new modification of the adaptive sliding-mode anti-synchronization scheme to study the adaptive anti-synchronization of different chaotic and hyperchaotic systems with unknown parameters. Lyapunov stability theory and the controller design establish the asymptotic stability of the anti-synchronization errors at the origin. Accordingly, suitable adaptive parameter update laws estimate the true values of uncertain parameters. Two numerical examples were used to provide illustrations and simulation results certified the performance of the proposed simple and generalized approach.

\section{Acknowledgement}

This research has been funded by Research Deanship of University of Ha'il Saudi Arabia through project number RG-191307

\section{References:}

1. Yang, X. J. (2012). Advanced Local Fractional Calculus and Its Applications; World Science Publisher,New York.

2. Al-sawalha, M. M. (2016). Anti-synchronization of fractional order chaotic and hyperchaotic systems with fully unknown parameters using modified adaptive control. Open Phys. (14), 304-313.

3. Hajipour, A.; Aminabadi, S. S. (2016). Synchronization of chaotic Arneodo system of incommensurate fractional order with unknown parameters using adaptive method. Optik, (127), 7704-7709.

4. Al-sawalha, M. M. (2017). Projective reduce order synchronization of fractional order chaotic systems with unknown parameters, J. Nonlinear Sci. (10), 2103-2114.

5. Deepika, D.; Sandeep, K.; Shiv, N. (2018). Uncertainty and disturbance estimator based robust synchronization for a class of uncertain fractional chaotic system via fractional order sliding mode control. Chaos, Solitons \& Fractals. (115), 196-203.

6. Tsung, C. L.; Tun. Y. L.; Valentina E. B. (2011) Adaptive fuzzy sliding mode control for synchronization of uncertain fractional order chaotic systems, Chaos, Solitons \& Fractals. (44), 791801

7. Ardashir. M. Z.; Sehraneh. G.; Okyay. K.; Sohrab. K. M. (2019). Robust predictive synchronization of uncertain fractional-order time-delayed chaotic systems. Soft Computing. (23), 6883-6898.

8. Ardashir. M. Z.; Sehraneh. G. (2017). Optimal synchronization of fractional-order chaotic systems subject to unknown fractional order, input nonlinearities and uncertain dynamic using type-2 fuzzy CMAC. Nonlinear Dynamics. (88), 2993-3002.

9. Ardashir. M. Z.; Sehraneh. G. (2018). Robust synchronization of uncertain fractional-order chaotic systems with time-varying delay. Nonlinear Dynamics. (93), 1809-1821.

10. Sakthivel. R.; Sakthivel. R.; Nithya. V.; Selvaraj. P.; Kwon. O. M. (2018). Fuzzy sliding mode control design of Markovian jump systems with time-varying delay. Journal of the Franklin Institute. (355), 6353-6370.

11. Selvaraj. P.; Kwon. O.M.; Sakthivel. R. (2019). Disturbance and uncertainty rejection performance for fractional-order complex dynamical networks. Neural Networks. (112), 73-84.

12. Agrawal, S. K.; Das, S. (2013). A modified adaptive control method for synchronization of some fractional chaotic systems with unknown parameters. Nonlinear Dynamics, (73), 907-919.

13. Pourmahmood. M., Khanmohammadi. S, Alizadeh. G, (2011). Synchronization of two different uncertain chaotic systems with unknown parameters using a robust adaptive sliding mode controller. Communications in Nonlinear Science and Numerical Simulation. (16), 2853-2868

14. Yahyazadeh. M, Noei. A. R, Ghaderi. R. (2011). Synchronization of chaotic systems with known and unknown parameters using a modified active sliding mode control. ISA Transactions, (50), 262-267.

15. Chen. X, Park J. H, Cao. J, , Qiu. J. (2017). Sliding mode synchronization of multiple chaotic systems with uncertainties and disturbances. Applied Mathematics and Computation. (308), 161-173.

16. Li. J, Li. W, Li, Q. (2012). Sliding mode control for uncertain chaotic systems with input nonlinearity, Communications in Nonlinear Science and Numerical Simulation. (17), 341-348.

17. Pourmahmood. M, Heydari. A. (2012). Chaos synchronization between two different chaotic systems with uncertainties, external disturbances, unknown parameters and input nonlinearities, Applied Mathematical Modelling, (36), 1639-1652.

18. Podlubny, I. ( 1999). Fractional Differential Equations; Academic Press: New York.

19. Liapunov, A. M. (1966). Stability of Motion; Elsevier/Academic Press, New York/London. 
On The Anti-Synchronization Of Fractional-Order Chaotic And Hyperchaotic Systems Via Modified Adaptive Sliding-Mode Control

20. Zhou, P.; Zhu, W. (2011). Function projective synchronization for fractional-order chaotic systems, Nonlinear Analysis: Real World Applications, (12), 811-816.

21. Lu, J. G; Chen, G. (2006). A note on the fractional-order Chen system. Chaos Solitons Fractals. (27), 685-688.

22. Li. T;Wang. Y; Yang. Y (2014). Synchronization of Fractional-Order Hyperchaotic Systems via Fractional-Order Controllers, Discrete Dynamics in Nature and Society. (2014) Article ID 408972. 